\title{
Clostridium cocleatum
}

National Cancer Institute

\section{Source}

National Cancer Institute. Clostridium cocleatum. NCI Thesaurus. Code C86278.

A species of anaerobic, Gram positive, rod shaped bacteria assigned to the phylum

Firmicutes. This species is urease and lipase negative, ferments cellobiose, fructose, galactose but not mannitol, hydrolyzes esculin, degrades mucin in the gastrointestinal tract and may appear helically coiled under the microscope. C. cocleatum is a component of the intestinal microflora but may provide a mechanism by which other bacteria can colonize and infect, through its mucin degrading properties. 